\title{
ON A CONVERSE RESULT FOR PERRON'S THEOREM FOR ASYMPTOTIC STABILITY FOR NONLINEAR DIFFERENTIAL EQUATIONS
}

\author{
GEORGE SEIFERT
}

\begin{abstract}
ABSTRACr. Two fairly simple proofs of a converse of Perron's classical theorem for the exponential asymptotic stability of the trivial solution of a nonlinear system of ordinary differential equations are given.
\end{abstract}

A well-known classical result, orginally due to Perron, giving a condition for asymptotic stability of the trivial solution of a system of ordinary differential equations may be stated as follows (cf. [1, Theorem 1.1, p. 314]): Suppose $A$ is an $n \times n$ matrix with complex constant entries. Let $x$ and $g(x, t)$ be $n$-vectors such that $g(x, t)$ is continuous for all $(x, t)$ and $g(x, t)=o(|x|)$ as $x \rightarrow 0$ uniformly for $t \geqslant 0$. Suppose all eigenvalues of $A$ have negative real parts. Then there exist positive constants $K, \delta$, and $\alpha$ such that if $x(t)$ is a solution of

$$
x^{\prime}=A x+g(x, t)
$$

such that $|x(0)| \leqslant \delta$, then $|x(t)| \leqslant K|x(0)| \exp (-\alpha t), t>0$.

What seems not as well known is that the converse of this result holds. The fact that it is necessary for the eigenvalues of $A$ to all have negative real parts if the conclusion of Perron's theorem as stated above holds will follow as a trivial corollary of the following theorem. In what follows $|x|$ and $|B|$ denote any compatible norms for the vector $x$ and matrix $B$ respectively.

THEOREM. Let $A$ and $g(x, t)$ be as above, and let there exist real-valued functions $\rho(r)$ and $\phi(t)$ defined and continuous on $[0, \delta]$ and $[0, \infty)$ respectively such that $\rho(r)=O(r)$ as $r \rightarrow 0+$ and $\phi(t) \rightarrow 0$ as $t \rightarrow \infty$, and such that if $x(t)$ is a solution of (1) with $|x(0)| \leqslant \delta$, then

$$
|x(t)| \leqslant \rho(|x(0)|) \phi(t) \quad \text { for } t>0 .
$$

Then all eigenvalues of $A$ have negative real parts.

Proof. Since our hypothesis implies $x=0$ is stable, all eigenvalues of $A$ must have nonpositive real parts. Assume one such eigenvalue is pure imaginary; we show that this leads to a contradiction.

Received by the editors February 10, 1986.

1980 Mathematics Subject Classification (1985 Revision). Primary 34D20.

(C1987 American Mathematical Society $0002-9939 / 87 \$ 1.00+\$ .25$ per page 
First without loss of generality we may suppose $A$ to be in Jordan canonical form, since any change of variable $x=P y$, when $P$ is an $n \times n$ nonsingular matrix, does not change the order condition on $g$ nor the hypotheses of the theorem. Thus

$$
A=\operatorname{diag}\left(J_{1}, J_{2}, \ldots, J_{m}\right) \text {, }
$$

where $J_{k}$ are the Jordan blocks; i.e. are $n_{k} \times n_{k}$ matrices of the form

$$
\left(\begin{array}{ccccc}
\lambda_{k} & 1 & 0 & \cdots & 0 \\
0 & \lambda_{k} & 1 & \cdots & 0 \\
\vdots & & \ddots & & \\
0 & \ldots & & 0 & \lambda_{k}
\end{array}\right) \quad \text { if } n_{k}>1
$$

and just the $1 \times 1$ matrix $\lambda_{k}$ if $n_{k}=1$. Here $\lambda_{k}$ are the eigenvalues of $A$ and we may suppose $\lambda_{1}=i \beta$.

A well-known result tells us that

$$
e^{t A}=\operatorname{diag}\left(e^{t J_{1}}, e^{t J_{2}}, \ldots, e^{t J_{m}}\right) .
$$

If $x_{0}=\left(\delta_{0}, 0, \ldots, 0\right), \delta_{0}>0$, it follows easily that

$$
\left|e^{t A} x_{0}\right|=\left|\delta_{0} e^{i \beta}\right|=\delta_{0}
$$

(cf. [1, pp. 74-77])

Now let $x(t)$ solve (1) with $x(0)=\left(\delta_{0}, 0, \ldots, 0\right), 0<\delta_{0} \leqslant \delta$. Using the familiar variation of constants formula, we obtain

$$
x(t)=e^{t A} x(0)+\int_{0}^{t} e^{(t-s) A} g(x(s), s) d s, \quad t \geqslant 0 .
$$

From our hypothesis on $g$, there exists a continuous function $h(x, t)$ such that $g(x, t)=|x| h(x, t)$ and $h(x, t) \rightarrow 0$ as $x \rightarrow 0$ uniformly for $t \geqslant 0$. So using (3) we get

$$
\left|e^{t A} x(0)\right| \leqslant|x(t)|+\int_{0}^{t}\left|e^{(t-s) A}\right||x(s)||h(x(s), s)| d s, \quad t \geqslant 0,
$$

which implies, using our hypotheses,

$$
\delta_{0} \leqslant \rho\left(\delta_{0}\right) \phi(t)+\rho\left(\delta_{0}\right) \int_{0}^{t}\left|e^{(t-s) A} \| h(x(s), s)\right| \phi(s) d s, \quad t \geqslant 0 .
$$

There exists a constant $M>0$ such that $\rho(r) \leqslant M r$ for $0 \leqslant r \leqslant \delta$. Fix $T>0$ so that

$$
\phi(T)<(3 M)^{-1}
$$

and $\delta_{1}, 0<\delta_{1} \leqslant \delta$, such that $|x| \leqslant \delta_{1}$ implies $|h(x, t)| \leqslant \varepsilon(T), t \geqslant 0$, where

$$
\varepsilon(T)=\left(3 M \int_{0}^{T}\left|e^{(T-s) A}\right| \phi(s) d s\right)^{-1} .
$$

Finally fix $\delta_{0}, 0<\delta_{0} \leqslant \delta$, such that

$$
\sup \left\{\rho\left(\delta_{0}\right) \phi(t): 0 \leqslant t \leqslant T\right\} \leqslant \delta_{1} .
$$

Put $t=T$ and $\delta_{0}$ as determined above into (4). Since $|x(s)| \leqslant \rho\left(\delta_{0}\right) \phi(s), 0 \leqslant s \leqslant T$, it follows that $|h(x(s), s)| \leqslant \varepsilon(T), 0 \leqslant s \leqslant T$, and so (4) implies

$$
1 \leqslant\left(\rho\left(\delta_{0}\right) / \delta_{0}\right)\left(\phi(T)+\varepsilon(T) \int_{0}^{T}\left|e^{(T-s) A}\right| \phi(s) d s\right) .
$$

Using (5) and (6) yields the contradiction $1 \leqslant \frac{2}{3}$. This completes the proof. 
Some remarks are in order. First, if $g(x, t)=g(x)$ in system (1), it can be shown directly that (2) implies the existence of positive constants $K$ and $\alpha$ such that for a solution $x(t)$ of (1) with $|x(0)|<\delta$,

$$
|x(t)| \leqslant K|x(0)| \exp (-\alpha t), \quad t>0 ;
$$

this was pointed out to the author by Professor George Sell. The fact that this is also true for systems (1) with nonautonomous $g$, where $g(x, t)=o(|x|)$ as $x \rightarrow 0$ uniformly for $t \geqslant 0$, follows since in this case (2) implies the conclusion of our theorem which in turn implies (E) (cf. [1, Theorem 1.1, p. 314]). It should be noted, however, that there are systems not of the form of (1) for which (2) holds and yet (E) does not; a simple example is the scalar equation $x^{\prime}=-x(1+t)^{-1}, t \geqslant 0$. It is also instructive to note that the solutions of scalar equation $x^{\prime}=-x^{3}$ which is of the form of (1) do not satisfy (2).

We include another proof of our theorem where instead of (2), we assume that $x=0$ is exponentially asymptotically stable in the standard sense. This condition is again equivalent to (2) for (1), and the proof is somewhat simpler.

We recall the standard definitions (cf. [2 or 3]). The solution $x=0$ of (1) is:

(a) stable if given $\varepsilon>0$ and $t_{0} \geqslant 0$, there exists a $\delta\left(\varepsilon, t_{0}\right)>0$ such that if $x(t)$ is a solution of (1) with $\left|x\left(t_{0}\right)\right|<\delta\left(\varepsilon, t_{0}\right)$, then $|x(t)|<\varepsilon$ for $t>t_{0}$,

(b) exponentially asymptotically stable if there exists an $\alpha>0$ and for each $\varepsilon>0$, a $\delta(\varepsilon)>0$ such that if $x(t)$ is a solution of (1) with $\left|x\left(t_{0}\right)\right|<\delta(\varepsilon)$, then $|x(t)|<$ $\varepsilon e^{-\alpha\left(t-t_{0}\right)}$ for $t>t_{0}$.

Suppose now $x=0$ is exponentially asymptotically stable for (1). With $\alpha>0$ as given in (b), consider the change of variables $y=e^{\alpha t} x$. It is easy to show that this takes (1) into

$$
y^{\prime}=A y-\alpha y+e^{\alpha t} g\left(e^{-\alpha t} y, t\right) \quad \text { or } \quad y^{\prime}=(A-\alpha I) y+|y| h\left(e^{-\alpha t} y, t\right),
$$

where $I$ is the $n \times n$ identity matrix, and $h$ is the function introduced in the proof of our theorem.

We now show that $y=0$ is stable for (7). To show this, let $\varepsilon>0$ be given. Then for any $t_{0} \geqslant 0$, there exists a $\delta\left(\varepsilon, t_{0}\right)>0$ such that for any solution $x(t)$ of (1) such that $\left|x\left(t_{0}\right)\right|<\delta\left(\varepsilon, t_{0}\right)$, we have

$$
|x(t)|<\left(\varepsilon e^{-\alpha t_{0}}\right) e^{-\alpha\left(t-t_{0}\right)}, \quad t>t_{0} ;
$$

i.e., $|x(t)|<\varepsilon e^{-\alpha t}, t>t_{0}$. Suppose $y(t)$ solves (7) with $\left|y\left(t_{0}\right)\right|<\delta\left(\varepsilon, t_{0}\right)$. This implies $e^{\alpha t_{0}}\left|x\left(t_{0}\right)\right|<\delta\left(\varepsilon, t_{0}\right)$; i.e., also $\left|x\left(t_{0}\right)\right|<\delta\left(\varepsilon, t_{0}\right)$. But then $|x(t)|<\varepsilon e^{-\alpha t}$, $t>t_{0}$; i.e., $e^{-\alpha t}|y(t)|<\varepsilon e^{-\alpha t}, t>t_{0}$, from which the stability of $y=0$ for (7) follows.

We now use a well-known result, already used in the proof of our theorem, which says that if $y=0$ is stable for $y^{\prime}=B y+f(y, t)$, where $B$ is an $n \times n$ matrix, and $f$ is continuous and satisfies $f(y, t)=o(|y|)$ as $y \rightarrow 0$ uniformly for $t \geqslant 0$, then all eigenvalues of $B$ have nonpositive real parts (cf. [1, p. 317]). Since (7) is of this form with $B=A-\alpha I$ and $f(y, t)=|y| h\left(e^{-\alpha t} y, t\right)$, it follows easily that all eigenvalues of $A$ must have negative real parts; in fact, have real parts less than $-\alpha$. 
Finally, as again pointed out by Professor George Sell, our theorem can be proved in case $g$ is Lipschitz in $x$ by using a result (Theorem 4) in [4]. Since this result is for systems like (1) with both $A$ and $g$ time-dependent and hence involves more detail than we need, we omit this proof, except to remark that the basic argument is as in our second proof.

The author wished to thank his colleague Professor S. N. S. Khalsa for suggesting the question of whether the converse of Perron's theorem holds.

\section{REFERENCES}

1. E. A. Coddington and N. Levinson, Theory of ordinary differential equations, McGraw-Hill, New York, 1955.

2. T. Yoshizawa, Stability theory by Lyapunov's second method, Math. Soc. Japan 9 (1966), 27-28.

3. H. Antosiewicz, A survey of Lyapunov's second method, Contributions to the Theory of Nonlinear Oscillations. IV, Ann. of Math. Studies, no. 41, Princeton Univ. Press, Princeton, N. J., 1958.

4. G. R. Sell, The structure of a flow in the vicinity of an almost periodic motion, J. Differential Equations 27 (1978), 359-393.

Department of Mathematics, Iowa State University, Ames, Iowa 50011 